\title{
ON DIVERSITY SCHEMES IN DECENTRALIZED ESTIMATION
}

\author{
Alex S. Leong and Subhrakanti Dey \\ Department of Electrical and Electronic Engineering \\ University of Melbourne, Vic. 3010, Australia \\ $\{$ asleong, sdey\}@unimelb.edu.au
}

\begin{abstract}
This paper is concerned with the decentralized estimation of a Gaussian source using multiple sensors. We consider a diversity scheme where only the sensor with the best channel sends their measurements to a fusion center, using the analog amplify and forwarding technique. A distributed version of the diversity scheme where sensors decide what to transmit based only on their local information is also considered. We derive asymptotic expressions for the expected distortion of these schemes as the number of sensors becomes large. We also study the optimal power allocation problem for minimizing the expected distortion subject to average power constraints.
\end{abstract}

\section{INTRODUCTION}

Wireless sensor networks have received much recent interest in the research community. Many different schemes for decentralized estimation of sources using multiple sensors have been proposed. One popular technique is analog amplify and forward [1], where sensors transmit a scaled version of their analog measurements to a fusion center, and has been shown to be optimal in some situations [2]. Analog forwarding under different multiple access schemes such as (coherent) multi-access [1,3] and orthogonal access [4], have been studied.

One problem with the analog amplify and forwarding technique is that it appears to be hard to implement, especially when the number of sensors is large (though studies suggest that even without e.g. perfect synchronization much of the gains can still be achieved [5]). This paper will study the performance of the analog forwarding technique using multiple access schemes which may be easier to implement, based on the concept of multi-user diversity [6,7]. For the problem of maximizing the sum rate subject to average power constraints, the optimal solution is to schedule the users such that at most only one user transmits, with this user being the one having the best channel conditions at that instance.

In this paper we will study the use of a similar diversity scheme in the decentralized estimation of a Gaussian source. In this scheme, which we will refer to as the multi-sensor diversity scheme, the sensor with the best channel conditions at that time will amplify and forward its measurement to the fusion center, while the other sensors do not transmit. A distributed version of the multi-sensor diversity scheme, similar to a distributed version of the multi-user diversity scheme studied in [8] called the channel-aware ALOHA scheme, will then also be considered.

In this paper we are interested in the asymptotic behaviour of such schemes as the number of sensors $M$ goes to infinity. It is

This work was supported by the Australian Research Council. shown that in many cases the expected distortion decays to a nonzero limit at the rate $1 / \ln (M)$. We will also be interested in deriving the optimal power allocation to minimize the expected distortion subject to average power constraints.

The paper is organised as follows. Section 2 specifies our model and the different multiple access schemes used by the sensors to communicate to the fusion center. Section 3 derives the asymptotic behaviour of these schemes, followed by comparisons and discussions. Optimal power allocation is considered in Section 4.

\section{SYSTEM MODEL}

We wish to estimate a discrete time signal $\theta_{k}$ modelled as an i.i.d. Gaussian source with zero mean and variance $\sigma_{\theta}^{2}$, with $k$ representing the time index. The Gaussian source is measured by $M$ sensors with sensor $i$ having measurements

$$
y_{i, k}=\theta_{k}+v_{i, k}, i=1, \ldots, M
$$

with $v_{i, k}$ being i.i.d. Gaussian with zero mean and noise variance $\sigma_{i}^{2}$, with $v_{i, k}$ independent of $v_{j, k}$ for $i \neq j$. Let $g_{i, k}$ be the randomly time-varying channel gains from sensor $i$ to the fusion center, and $\alpha_{i, k}$ the amplification factors in the amplify and forward scheme. We assume that $g_{i, k}$ and $g_{j, k}$ are independent for $i \neq j$. The transmit power of sensor $i$ at time $k$ is defined as

$$
\gamma_{i, k}=\alpha_{i, k}^{2} \mathbb{E}\left[y_{i, k}^{2}\right]=\alpha_{i, k}^{2}\left(\sigma_{\theta}^{2}+\sigma_{i}^{2}\right)
$$

Different multiple access schemes for transmitting the sensor measurements to a fusion center will now be presented.

\subsection{Multi-sensor diversity scheme}

Let $g_{\max , k}=\max \left(g_{1, k}, \ldots, g_{M, k}\right)$, and $i^{*}$ the index of the corresponding sensor. Consider a scheme where only the sensor with the best channel transmits its measurement to the fusion center. The fusion center then receives

$$
z_{k}=\sqrt{g_{\max , k}} \alpha_{i^{*}, k}\left(\theta_{k}+v_{i^{*}, k}\right)+n_{k}
$$

where $n_{k}$ is i.i.d. Gaussian with zero mean and variance $\sigma_{n}^{2}$. Using the linear MMSE estimator, the mean squared error or distortion at time $k$ can be easily shown to be

$$
D_{k}=\left(\frac{1}{\sigma_{\theta}^{2}}+\frac{g_{\max , k} \alpha_{i^{*}, k}^{2}}{g_{\max , k} \alpha_{i^{*}, k}^{2} \sigma_{i^{*}}^{2}+\sigma_{n}^{2}}\right)^{-1}
$$




\subsection{Channel-aware ALOHA scheme}

The multi-sensor diversity scheme requires knowledge of all the channel gains in order to determine the sensor with the best channel. We consider now a scheme that we will call the channel-aware ALOHA scheme, that is based on a distributed scheme for multi-user diversity studied in [8], see also [9] for a similar scheme in the distributed estimation of a constant parameter. In this scheme a sensor will forward its measurement to the fusion center only if $g_{i, k}>T_{i}$ for some threshold $T_{i}$.

In [8], choosing $T_{i}$ such that $\operatorname{Pr}\left(g_{i, k}>T_{i}\right)=1 / M, \forall i$, was shown to be asymptotically optimal, in the sense that this gives the same rate of throughput scaling as in the multi-user diversity scheme, but with a fraction of throughput loss of $1 / e$. In this paper we will also use this choice of $T_{i}$.

In this scheme, if more than one sensor transmits, then a collision is assumed and $D_{k}=\sigma_{\theta}^{2}$. Similarly, if no sensor transmits then $D_{k}=\sigma_{\theta}^{2}$. If only one sensor transmits, then

$$
D_{k}=\left(\frac{1}{\sigma_{\theta}^{2}}+\frac{g_{i^{*}, k} \alpha_{i^{*}, k}^{2}}{g_{i^{*}, k} \alpha_{i^{*}, k}^{2} \sigma_{i^{*}}^{2}+\sigma_{n}^{2}}\right)^{-1}
$$

where $i^{*}$ is the index of the sensor that is transmitting.

\section{ASYMPTOTIC ANALYSIS}

We are interested in deriving asymptotic expressions for $\mathbb{E}\left[D_{k}\right]$ as $M \rightarrow \infty$, where the expectation is over the random channel gains $g_{i, k}$, for the diversity schemes in Section 2. By the i.i.d. (in time) nature of the models we will drop the subscript $k$. For analytical tractibility we will first analyze "symmetric" sensor networks with $\sigma_{i}^{2}=\sigma_{v}^{2}, \forall i$, with the $g_{i}$ 's being identically distributed, and simple power allocation policies. See Section 3.4 for remarks on more general asymmetric situations and Section 4 for optimal power allocation. In order to obtain precise asymptotic results we will need to assume a specific distribution. In this paper we will assume Rayleigh fading, though most of our analytical methods should be able to be adapted to other fading distributions.

Notation: For two functions $f(t)$ and $g(t)$, we will use the standard asymptotic notation (see e.g. [10]) and say that $f \sim g$ as $t \rightarrow t_{0}$, if $\frac{f(t)}{g(t)} \rightarrow 1$ as $t \rightarrow t_{0}$. It is well known that the asymptotic relation $\sim$ is retained under addition, multiplication and division.

\subsection{Multi-sensor diversity scheme}

Let us use $\alpha_{i^{*}}=1$, and $\alpha_{j}=0, \forall j \neq i^{*}$. Then

$$
\begin{aligned}
D & =\left(\frac{1}{\sigma_{\theta}^{2}}+\frac{g_{\max }}{g_{\max } \sigma_{v}^{2}+\sigma_{n}^{2}}\right)^{-1} \\
& =\frac{\sigma_{\theta}^{2} \sigma_{v}^{2}}{\sigma_{\theta}^{2}+\sigma_{v}^{2}}\left(1+\frac{\sigma_{n}^{2} \sigma_{\theta}^{2}}{\sigma_{v}^{2}\left(\sigma_{\theta}^{2}+\sigma_{v}^{2}\right)} \frac{1}{g_{\max }+\frac{\sigma_{n}^{2}}{\sigma_{\theta}^{2}+\sigma_{v}^{2}}}\right)
\end{aligned}
$$

Considering Rayleigh fading, we have the following result.

Lemma 1. Suppose the $g_{i}$ 's are exponentially distributed with mean $1 / \lambda$, and let $b>0$ be a constant. Then

$$
\mathbb{E}\left[\frac{1}{g_{\max }+b}\right] \sim \frac{\lambda}{\lambda b+\ln M} \sim \frac{\lambda}{\ln (M)} \text { as } M \rightarrow \infty
$$

See [11] for the proof of Lemma 1. By Lemma 1, we then have

$$
\begin{aligned}
\mathbb{E}[D] & \sim \frac{\sigma_{\theta}^{2} \sigma_{v}^{2}}{\sigma_{\theta}^{2}+\sigma_{v}^{2}}\left[1+\frac{\sigma_{n}^{2} \sigma_{\theta}^{2}}{\sigma_{v}^{2}\left(\sigma_{\theta}^{2}+\sigma_{v}^{2}\right)} \frac{\lambda}{\ln (M)+\frac{\lambda \sigma_{n}^{2}}{\sigma_{\theta}^{2}+\sigma_{v}^{2}}}\right] \\
& \sim \frac{\sigma_{\theta}^{2} \sigma_{v}^{2}}{\sigma_{\theta}^{2}+\sigma_{v}^{2}}\left[1+\frac{\sigma_{n}^{2} \sigma_{\theta}^{2}}{\sigma_{v}^{2}\left(\sigma_{\theta}^{2}+\sigma_{v}^{2}\right)} \frac{\lambda}{\ln (M)}\right]
\end{aligned}
$$

Hence as $M \rightarrow \infty$, the expected distortion goes to $\frac{\sigma_{\theta}^{2} \sigma_{v}^{2}}{\sigma_{\theta}^{2}+\sigma_{v}^{2}}$ at the rate $1 / \ln (M)$.

\subsection{Channel-aware ALOHA scheme}

Recall that $T_{i}$ is chosen such that $\operatorname{Pr}\left(g_{i}>T_{i}\right)=1 / M$. Let us use $\alpha_{i}=1$ if sensor $i$ transmits. By the symmetry of the situation $T_{i}=T, \forall i$. Note that by the choice of $T$ each sensor has probability $1 / M$ of transmitting to the fusion center (some of which will result in collision), so the long term average power usage is the same as in the multi-sensor diversity scheme. Then

$$
\begin{aligned}
& \operatorname{Pr}(\text { no sensor transmits })=\left(\operatorname{Pr}\left(g_{i}<T\right)\right)^{M}=\left(1-\frac{1}{M}\right)^{M} \\
& \operatorname{Pr} \text { (successful transmission) } \\
& =M \operatorname{Pr}(\text { sensor } i \text { transmits successfully) } \\
& =M \operatorname{Pr}\left(g_{i}>T\right) \prod_{j \neq i} \operatorname{Pr}\left(g_{j}<T\right) \\
& =M \frac{1}{M}\left(1-\frac{1}{M}\right)^{M-1}=\left(1-\frac{1}{M}\right)^{M-1} \\
& \operatorname{Pr}(\text { collision })=1-\left(1-\frac{1}{M}\right)^{M}-\left(1-\frac{1}{M}\right)^{M-1}
\end{aligned}
$$

We have

$$
\begin{aligned}
& \mathbb{E}[D]=\sigma_{\theta}^{2} \operatorname{Pr}(\text { no sensor transmits })+\sigma_{\theta}^{2} \operatorname{Pr} \text { (collision) } \\
&+ \frac{1}{\operatorname{Pr}\left(g_{i}>T\right)} \int_{T}^{\infty}\left(\frac{1}{\sigma_{\theta}^{2}}+\frac{g_{i}}{g_{i} \sigma_{v}^{2}+\sigma_{n}^{2}}\right)^{-1} p\left(g_{i}\right) d g_{i} \\
& \times \operatorname{Pr}(\text { successful transmission) } \\
&=\sigma_{\theta}^{2}\left[1-\left(1-\frac{1}{M}\right)^{M-1}\right]+M\left(1-\frac{1}{M}\right)^{M-1} \\
& \quad \times \int_{T}^{\infty} \frac{\sigma_{\theta}^{2} \sigma_{v}^{2}}{\sigma_{\theta}^{2}+\sigma_{v}^{2}}\left(1+\frac{\sigma_{n}^{2} \sigma_{\theta}^{2}}{\sigma_{v}^{2}\left(\sigma_{\theta}^{2}+\sigma_{v}^{2}\right)} \frac{1}{g_{i}+\frac{\sigma_{n}^{2}}{\sigma_{\theta}^{2}+\sigma_{v}^{2}}}\right) p\left(g_{i}\right) d g_{i}
\end{aligned}
$$

Now assume that the $g_{i}$ 's are exponentially distributed with mean $1 / \lambda$. Then $T=\frac{1}{\lambda} \ln M$ and

$$
\int_{T}^{\infty} \frac{1}{g+b} \lambda \exp (-\lambda g) d g=\lambda \exp (\lambda b) E_{1}(\lambda(b+T))
$$

Hence

$$
\begin{aligned}
& \mathbb{E}[D]=\sigma_{\theta}^{2}\left[1-\left(1-\frac{1}{M}\right)^{M-1}\right]+M\left(1-\frac{1}{M}\right)^{M-1} \frac{\sigma_{\theta}^{2} \sigma_{v}^{2}}{\sigma_{\theta}^{2}+\sigma_{v}^{2}} \\
& \times\left[\frac{1}{M}+\frac{\lambda \sigma_{n}^{2} \sigma_{\theta}^{2}}{\sigma_{v}^{2}\left(\sigma_{\theta}^{2}+\sigma_{v}^{2}\right)} \exp \left(\frac{\lambda \sigma_{n}^{2}}{\sigma_{\theta}^{2}+\sigma_{v}^{2}}\right) E_{1}\left(\frac{\lambda \sigma_{n}^{2}}{\sigma_{\theta}^{2}+\sigma_{v}^{2}}+\ln M\right)\right] \\
& \sim \sigma_{\theta}^{2}\left(1-\frac{1}{e}\right)+\frac{1}{e} \frac{\sigma_{\theta}^{2} \sigma_{v}^{2}}{\sigma_{\theta}^{2}+\sigma_{v}^{2}}\left[1+\frac{\sigma_{n}^{2} \sigma_{\theta}^{2}}{\sigma_{v}^{2}\left(\sigma_{\theta}^{2}+\sigma_{v}^{2}\right)} \frac{\lambda}{\ln M+\frac{\lambda \sigma_{n}^{2}}{\sigma_{\theta}^{2}+\sigma_{v}^{2}}}\right] \\
& \sim \sigma_{\theta}^{2}\left(1-\frac{1}{e}\right)+\frac{1}{e} \frac{\sigma_{\theta}^{2} \sigma_{v}^{2}}{\sigma_{\theta}^{2}+\sigma_{v}^{2}}\left[1+\frac{\sigma_{n}^{2} \sigma_{\theta}^{2}}{\sigma_{v}^{2}\left(\sigma_{\theta}^{2}+\sigma_{v}^{2}\right)} \frac{\lambda}{\ln M}\right]
\end{aligned}
$$


as $M \rightarrow \infty$, where we have used the asymptotic expansion $E_{1}(z) \sim$ $\frac{e^{-z}}{z}\left(1-\frac{1}{z}+\frac{2}{z^{2}}-+\ldots\right)$. The expected distortion in this case goes to $\sigma_{\theta}^{2}\left(1-\frac{1}{e}\right)+\frac{1}{e} \frac{\sigma_{\theta}^{2} \sigma_{v}^{2}}{\sigma_{\theta}^{2}+\sigma_{v}^{2}}$ at the rate $1 / \ln (M)$ as $M \rightarrow \infty$.

\subsection{Comparisons and discussions}

The limit $\frac{\sigma_{\theta}^{2} \sigma_{v}^{2}}{\sigma_{\theta}^{2}+\sigma_{v}^{2}}=\left(\frac{1}{\sigma_{\theta}^{2}}+\frac{1}{\sigma_{v}^{2}}\right)^{-1}$ in the multi-sensor diversity scheme corresponds to the distortion that can be achieved with a single sensor, with estimation performed at that sensor, i.e. no further analog forwarding to a fusion center. For the channel-aware ALOHA scheme, the limit is $\sigma_{\theta}^{2}\left(1-\frac{1}{e}\right)+\frac{1}{e} \frac{\sigma_{\theta}^{2} \sigma_{v}^{2}}{\sigma_{\theta}^{2}+\sigma_{v}^{2}}$, which is clearly larger than the limit in the multi-sensor diversity scheme. It can be regarded as a weighted combination of the limiting value $\frac{\sigma_{\theta}^{2} \sigma_{v}^{2}}{\sigma_{\theta}^{2}+\sigma_{v}^{2}}$ when there is a successful transmission, and the distortion $\sigma_{\theta}^{2}$ when transmissions are unsuccessful, with $\frac{1}{e}$ being the asymptotic probability of successful transmission as $M \stackrel{e}{\rightarrow} \infty$ (which also corresponds to the asymptotic throughput of a slotted ALOHA system [8]).

In terms of speed of convergence, the rate of $1 / \ln (M)$ is achieved in the diversity schemes. A similar $1 / \ln (M)$ rate is achieved when sensor measurements are transmitted to a fusion center digitally using separate source/channel coding, e.g. as in the CEO problem $[1,12]$. In contrast, a rate of $1 / M$ has been shown for the coherent multi-access and orthogonal access schemes, see e.g. $[1,4,11]$, though as stated in the introduction, such schemes may be harder to implement in practice.

\subsection{General parameters}

Here we will briefly describe how the results of Section 3 change when the sensor noise variances are not necessarily identical, and the fading channels are not necessarily identically distributed. The full details can be found in [11]. The idea is to obtain upper and lower bounds on the expected distortion which asymptotically will have the same scaling behaviour, a similar method was used in [13] in the context of linear state estimation.

In the case where the sensor noise variances $\sigma_{i}^{2}, i=1, \ldots, M$ are not necessarily identical but satisfy $0<\sigma_{\min }^{2} \leq \sigma_{i}^{2} \leq \sigma_{\max }^{2}<$ $\infty, \forall i$, and the fading channels are i.i.d. across sensors, it turns out that in both the multi-sensor diversity and channel-aware ALOHA schemes the upper and lower bounds do not converge to the same limit as $M \rightarrow \infty$, so for general sensor noise variances one can not say much more about its asymptotic behaviour.

We also consider the case where the sensor noise variances are identical $\left(\sigma_{i}^{2}=\sigma_{v}^{2}, \forall i\right)$, and the fading channels are independent but not necessarily identically distributed, satisfying the assumption that the channel gains $g_{i}$ can be written as $g_{i}=\mu_{i} h_{i}, \forall i$, where $\mu_{i}>0$ are constants satisfying $0<\mu_{\min } \leq \mu_{i} \leq \mu_{\max }<\infty$, and the $h_{i}$ 's are identically distributed. It turns out that in this case the scaling behaviour will be preserved, in the sense that the upper and lower bounds derived will both converge to the same limit at the same rate.

\subsection{Numerical studies}

Consider a situation with $\sigma_{\theta}^{2}=1, \sigma_{v}^{2}=0.2, \sigma_{n}^{2}=0.1$, and let $g_{i}, \forall i$ be exponentially distributed with mean $1 / 2$. Note that then $\left(\frac{1}{\sigma_{\theta}^{2}}+\frac{1}{\sigma_{v}^{2}}\right)^{-1}=0.1667$ and $\sigma_{\theta}^{2}\left(1-\frac{1}{e}\right)+\frac{1}{e} \frac{\sigma_{\theta}^{2} \sigma_{v}^{2}}{\sigma_{\theta}^{2}+\sigma_{v}^{2}}=0.6934$.

In Fig.1(a) we compare between the simulated expected distortion (averaging over 100000 iterations) and the asymptotic expres- sion (1) for the multi-sensor diversity scheme, for different numbers of sensors $M$. In Fig.1(b) we compare between the simulated expected distortion and the asymptotic expression (2) for the channelaware ALOHA scheme, for different numbers of sensors $M$. In each case, the validity of the respective asymptotic expressions for large $M$ is confirmed. We also see that in the channel-aware ALOHA scheme, the expected distortion is not necessarily monotonically decreasing with the number of sensors, though for large $M$ the $1 / \ln (M)$ decay will still occur.
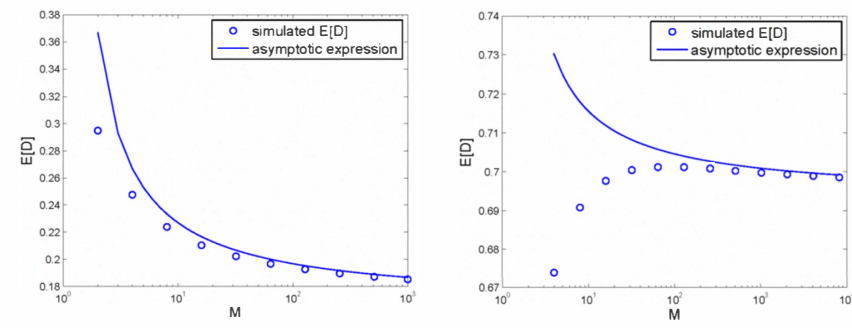

(a) Multi-sensor diversity scheme (b) Channel-aware ALOHA scheme

Fig. 1. Comparison between simulated expected distortion and asymptotic expression.

\section{OPTIMAL POWER ALLOCATION}

In this section we consider optimal power allocation for the multisensor diversity and channel-aware ALOHA schemes. For notational simplicity, and since we are also interested in the performance using optimal allocation for large numbers of sensors, we will consider symmetric sensor networks, though the results can be generalized to general parameters.

\subsection{Multi-sensor diversity scheme}

We are interested in minimizing the expected distortion subject $E[D]$ to an average power constraint $\mathcal{P}$. For the multi-sensor diversity scheme we can write this as

$$
\begin{aligned}
& \min _{\alpha_{i^{*}}^{2}} \frac{\sigma_{\theta}^{2} \sigma_{v}^{2}}{\sigma_{\theta}^{2}+\sigma_{v}^{2}} \mathbb{E}\left[1+\frac{\sigma_{n}^{2} \sigma_{\theta}^{2}}{\sigma_{v}^{2}\left(\sigma_{\theta}^{2}+\sigma_{v}^{2}\right)} \frac{1}{g_{\max } \alpha_{i^{*}}^{2}+\frac{\sigma_{n}^{2}}{\sigma_{\theta}^{2}+\sigma_{v}^{2}}}\right] \\
& \text { s.t. } \mathbb{E}\left[\alpha_{i^{*}}^{2}\right] \leq \frac{\mathcal{P}}{\sigma_{\theta}^{2}+\sigma_{v}^{2}}
\end{aligned}
$$

We use the following result:

Lemma 2. Consider the following problem

$$
\min _{\alpha_{i^{*}}^{2}} \mathbb{E}\left[\frac{1}{g_{\max } \alpha_{i^{*}}^{2}+b}\right] \text { s.t. } \mathbb{E}\left[\alpha_{i^{*}}^{2}\right] \leq \frac{\mathcal{P}}{\sigma_{\theta}^{2}+\sigma_{v}^{2}}
$$

The optimal solution is of the form

$$
\alpha_{i^{*}}^{2}=\left\{\begin{array}{ccc}
\sqrt{\frac{1}{g_{\max \nu}}-\frac{b}{g_{\max }}} & , \quad g_{\max } \geq b^{2} \nu \\
0 & , & \text { otherwise }
\end{array}\right.
$$

where $\nu$ satisfies

$$
\int_{b^{2} \nu}^{\infty}\left(\sqrt{\frac{1}{g_{\max } \nu}}-\frac{b}{g_{\max }}\right) p\left(g_{\max }\right) d g_{\max }=\frac{\mathcal{P}}{\sigma_{\theta}^{2}+\sigma_{v}^{2}}
$$


Proof. The derivation uses similar techniques to the capacity maximization problems for fading channels in $[6,14]$, and is omitted for brevity.

Using Lemma 2, the optimal power allocation for problem (3) is given by (4), with $b=\frac{\sigma_{n}^{2}}{\sigma_{\theta}^{2}+\sigma_{v}^{2}}$. The expected distortion under optimal power allocation can be computed as

$$
\begin{aligned}
\mathbb{E}[D] & =\int_{b^{2} \nu}^{\infty} \frac{\sigma_{\theta}^{2} \sigma_{v}^{2}}{\sigma_{\theta}^{2}+\sigma_{v}^{2}}\left(1+\frac{\sigma_{n}^{2} \sigma_{\theta}^{2}}{\sigma_{v}^{2}\left(\sigma_{\theta}^{2}+\sigma_{v}^{2}\right)} \sqrt{\frac{\nu}{g_{\max }}}\right) p\left(g_{\max }\right) d g_{\max } \\
& +\int_{0}^{b^{2} \nu} \sigma_{\theta}^{2} p\left(g_{\max }\right) d g_{\max }
\end{aligned}
$$

where $\nu$ satisfies (5), and can be determined numerically.

\subsection{Channel-aware ALOHA scheme}

For the channel-aware ALOHA scheme, the problem of minimizing the expected distortion subject $E[D]$ to an average power constraint $\mathcal{P}$ can be written as

$$
\begin{aligned}
& \min _{\alpha_{i}^{2}} \sigma_{\theta}^{2}\left[1-\left(1-\frac{1}{M}\right)^{M-1}\right]+M\left(1-\frac{1}{M}\right)^{M-1} \\
& \quad \times \int_{T}^{\infty} \frac{\sigma_{\theta}^{2} \sigma_{v}^{2}}{\sigma_{\theta}^{2}+\sigma_{v}^{2}}\left(1+\frac{\sigma_{n}^{2} \sigma_{\theta}^{2}}{\sigma_{v}^{2}\left(\sigma_{\theta}^{2}+\sigma_{v}^{2}\right)} \frac{1}{\alpha_{i}^{2} g_{i}+\frac{\sigma_{n}^{2}}{\sigma_{\theta}^{2}+\sigma_{v}^{2}}}\right) p\left(g_{i}\right) d g_{i} \\
& \text { s.t. } M \int_{T}^{\infty} \alpha_{i}^{2} p\left(g_{i}\right) d g_{i} \leq \frac{\mathcal{P}}{\sigma_{\theta}^{2}+\sigma_{v}^{2}}
\end{aligned}
$$

Similar to the multi-sensor diversity scheme, we have the following result:

Lemma 3. Consider the following problem

$\min _{\alpha_{i}^{2}} \int_{T}^{\infty} \frac{1}{g_{i} \alpha_{i}^{2}+b} p\left(g_{i}\right) d g_{i}$ s.t. $\int_{T}^{\infty} \alpha_{i}^{2} p\left(g_{i}\right) d g_{i} \leq \frac{\mathcal{P}}{M\left(\sigma_{\theta}^{2}+\sigma_{v}^{2}\right)}$

The optimal solution is of the form

$$
\alpha_{i}^{2}=\left\{\begin{array}{ccc}
\sqrt{\frac{1}{g_{i} \nu}}-\frac{b}{g_{i}} & , & g_{i} \geq \max \left(T, b^{2} \nu\right) \\
0 & , & \text { otherwise }
\end{array}\right.
$$

where $\nu$ satisfies

$$
\int_{\max \left(T, b^{2} \nu\right)}^{\infty}\left(\sqrt{\frac{1}{g_{i} \nu}}-\frac{b}{g_{i}}\right) p\left(g_{i}\right) d g_{i}=\frac{\mathcal{P}}{M\left(\sigma_{\theta}^{2}+\sigma_{v}^{2}\right)}
$$

Using Lemma 3, the optimal power allocation for problem (7) is given by (8), with $b=\frac{\sigma_{n}^{2}}{\sigma_{\theta}^{2}+\sigma_{v}^{2}}$. The expected distortion under optimal power allocation can be computed as

$$
\begin{aligned}
\mathbb{E}[D]= & \sigma_{\theta}^{2}\left[1-\left(1-\frac{1}{M}\right)^{M-1}\right]+M\left(1-\frac{1}{M}\right)^{M-1} \\
& \times \int_{\max \left(T, b^{2} \nu\right)}^{\infty} \frac{\sigma_{\theta}^{2} \sigma_{v}^{2}}{\sigma_{\theta}^{2}+\sigma_{v}^{2}}\left(1+\frac{\sigma_{n}^{2} \sigma_{\theta}^{2}}{\sigma_{v}^{2}\left(\sigma_{\theta}^{2}+\sigma_{v}^{2}\right)} \sqrt{\frac{\nu}{g_{i}}}\right) p\left(g_{i}\right) d g_{i} \\
& +M\left(1-\frac{1}{M}\right)^{M-1} \int_{T}^{\max \left(T, b^{2} \nu\right)} \sigma_{\theta}^{2} p\left(g_{i}\right) d g_{i}
\end{aligned}
$$

where $\nu$ satisfies (9).

\subsection{Numerical studies}

We again consider a situation with $\sigma_{\theta}^{2}=1, \sigma_{v}^{2}=0.2, \sigma_{n}^{2}=0.1$, and let $g_{i}, \forall i$ be exponentially distributed with mean $1 / 2$. For a fair comparison, when performing optimal power allocation we will take $\mathcal{P} /\left(\sigma_{\theta}^{2}+\sigma_{v}^{2}\right)=1$.

In Fig.2(a) we plot the expected distortion under constant (when the sensor is transmitting) and optimal power allocation, for the multisensor diversity scheme with different numbers of sensors. In Fig.2(b) we plot the expected distortion under constant and optimal power allocation, for the channel-aware ALOHA scheme with different numbers of sensors. The performance using constant power allocation can be seen to be very close to the performance under optimal power allocation, particularly for large numbers of sensors.
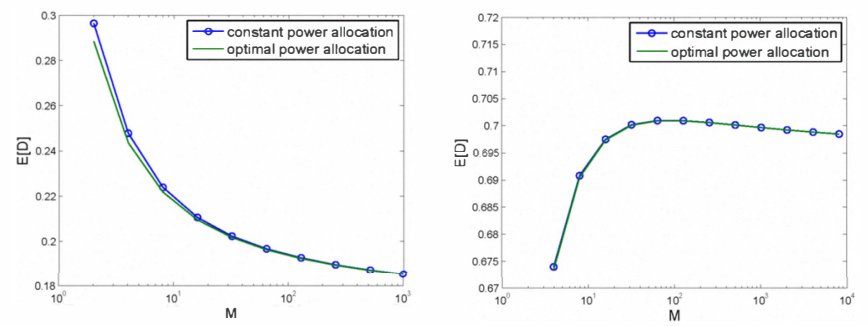

(a) Multi-sensor diversity scheme (b) Channel-aware ALOHA scheme

Fig. 2. Comparison between constant and optimal power allocation.

\subsection{Asymptotic behaviour under optimal power allocation}

In this subsection we will prove why the optimal power allocation and constant power allocation schemes perform so close to each other. We will assume that $g_{i}$ are exponentially distributed with mean $1 / \lambda$. We will also take $\mathcal{P} /\left(\sigma_{\theta}^{2}+\sigma_{v}^{2}\right)=1$.

\subsubsection{Multi-sensor diversity scheme}

Theorem 4. For the multi-sensor diversity scheme under optimal power allocation, as $M \rightarrow \infty$,

$$
\mathbb{E}[D] \sim \frac{\sigma_{\theta}^{2} \sigma_{v}^{2}}{\sigma_{\theta}^{2}+\sigma_{v}^{2}}\left[1+\frac{\sigma_{n}^{2} \sigma_{\theta}^{2}}{\sigma_{v}^{2}\left(\sigma_{\theta}^{2}+\sigma_{v}^{2}\right)} \frac{\lambda}{\ln (M)}\right]
$$

Proof. Firstly, by using similar techniques to the proof of Lemma 1, we can derive that

$$
\int_{0}^{\infty} \frac{1}{\sqrt{x}} M\left(1-e^{-\lambda x}\right)^{M-1} \lambda e^{-\lambda x} d x \sim \sqrt{\frac{\lambda}{\ln (M)}}
$$

and

$$
\int_{0}^{\infty} \frac{1}{x} M\left(1-e^{-\lambda x}\right)^{M-1} \lambda e^{-\lambda x} d x \sim \frac{\lambda}{\ln (M)}
$$

We have the following result:

Lemma 5. $\nu \rightarrow 0$ as $M \rightarrow \infty$, where $\nu$ satisfies (5).

See Appendix for the proof of Lemma 5. By Lemma 5 and (11)(12), the condition

$$
\int_{b^{2} \nu}^{\infty}\left(\sqrt{\frac{1}{g_{\max } \nu}}-\frac{b}{g_{\max }}\right) p\left(g_{\max }\right) d g_{\max }=1
$$


is asymptotically

$$
\sqrt{\frac{\lambda}{\nu \ln (M)}}-\frac{b \lambda}{\ln (M)} \sim 1
$$

We can easily solve for $\nu$ to get

$$
\nu \sim \frac{\lambda}{\ln (M)} \frac{1}{1+\frac{2 b \lambda}{\ln (M)}+\left(\frac{b \lambda}{\ln (M)}\right)^{2}} \sim \frac{\lambda}{\ln (M)}
$$

and so

$$
\int_{b^{2} \nu}^{\infty} \sqrt{\frac{\nu}{x}} M\left(1-e^{-\lambda x}\right)^{M-1} \lambda e^{-\lambda x} d x \sim \sqrt{\frac{\nu \lambda}{\ln (M)}} \sim \frac{\lambda}{\ln (M)}
$$

We also note that

$$
\left(1-e^{-\lambda b^{2} \nu}\right)^{M}=\left(1-\exp \left(-\frac{\lambda^{2} b^{2}}{\ln (M)}\right)\right)^{M}=o\left(\frac{1}{\ln (M)}\right)
$$

Hence from (6),

$$
\begin{aligned}
\mathbb{E}[D] & \sim \frac{\sigma_{\theta}^{2} \sigma_{v}^{2}}{\sigma_{\theta}^{2}+\sigma_{v}^{2}}\left[1+\frac{\sigma_{n}^{2} \sigma_{\theta}^{2}}{\sigma_{v}^{2}\left(\sigma_{\theta}^{2}+\sigma_{v}^{2}\right)} \frac{\lambda}{\ln (M)}\right]+\sigma_{\theta}^{2}\left(1-e^{-\lambda b^{2} \nu}\right)^{M} \\
& \sim \frac{\sigma_{\theta}^{2} \sigma_{v}^{2}}{\sigma_{\theta}^{2}+\sigma_{v}^{2}}\left[1+\frac{\sigma_{n}^{2} \sigma_{\theta}^{2}}{\sigma_{v}^{2}\left(\sigma_{\theta}^{2}+\sigma_{v}^{2}\right)} \frac{\lambda}{\ln (M)}\right]
\end{aligned}
$$

which is the same asymptotic expression as (1) of Section 3.

\subsubsection{Channel-aware ALOHA scheme}

Theorem 6. For the channel-aware ALOHA scheme under optimal power allocation, as $M \rightarrow \infty$,

$$
\mathbb{E}[D] \sim \sigma_{\theta}^{2}\left(1-\frac{1}{e}\right)+\frac{1}{e} \frac{\sigma_{\theta}^{2} \sigma_{v}^{2}}{\sigma_{\theta}^{2}+\sigma_{v}^{2}}\left[1+\frac{\sigma_{n}^{2} \sigma_{\theta}^{2}}{\sigma_{v}^{2}\left(\sigma_{\theta}^{2}+\sigma_{v}^{2}\right)} \frac{\lambda}{\ln M}\right]
$$

Due to space constraints, the proof is omitted but can be found in [11]. It uses techniques similar to the proof of Theorem 4.

\section{APPENDIX}

Proof. The proof is by contradiction. Regard $\nu(M)$ as a function of $M$. Suppose $\nu(M)$ does not converge to 0 as $M \rightarrow \infty$. Then there exists a constant $\bar{\nu}>0$ such that $\nu(M) \geq \bar{\nu}$ for infinitely many values of $M$. In particular, there are infinitely many values of $M$ such that the following is true:

$$
\begin{aligned}
\int_{b^{2} \nu(M)}^{\infty} & \sqrt{\frac{1}{x \nu(M)}} M\left(1-e^{-\lambda x}\right)^{M-1} \lambda e^{-\lambda x} d x \\
& \leq \frac{1}{\sqrt{\bar{\nu}}} \int_{b^{2} \bar{\nu}}^{\infty} \frac{1}{\sqrt{x}} M\left(1-e^{-\lambda x}\right)^{M-1} \lambda e^{-\lambda x} d x \\
& <\frac{1}{\sqrt{\bar{\nu}}} \int_{0}^{\infty} \frac{1}{\sqrt{x}} M\left(1-e^{-\lambda x}\right)^{M-1} \lambda e^{-\lambda x} d x \\
& \sim \sqrt{\frac{\lambda}{\bar{\nu} \ln (M)}}
\end{aligned}
$$

where the last line comes from (11). Since

$$
\int_{b^{2} \nu(M)}^{\infty} \frac{b}{x} M\left(1-e^{-\lambda x}\right)^{M-1} \lambda e^{-\lambda x} d x \geq 0,
$$

the condition

$$
\int_{b^{2} \nu(M)}^{\infty}\left(\sqrt{\frac{1}{g_{\max } \nu(M)}}-\frac{b}{g_{\max }}\right) p\left(g_{\max }\right) d g_{\max }=1
$$

thus cannot be satisfied for all $M$, which is a contradiction.

\section{REFERENCES}

[1] M. Gastpar and M. Vetterli, "Source-channel communication in sensor networks," Springer Lecture Notes in Computer Science, vol. 2634, pp. 162-177, Apr. 2003.

[2] M. Gastpar, "Uncoded transmission is exactly optimal for a simple Gaussian sensor network," IEEE Trans. Inform. Theory, vol. 54, no. 11, pp. 5247-5251, Nov. 2008.

[3] J.-J. Xiao, S. Cui, Z.-Q. Luo, and A. J. Goldsmith, "Linear coherent decentralized estimation," IEEE Trans. Signal Processing, vol. 56, no. 2, pp. 757-770, Feb. 2008.

[4] S. Cui, J.-J. Xiao, A. Goldsmith, Z.-Q. Luo, and H. V. Poor, "Estimation diversity and energy efficiency in distributed sensing," IEEE Trans. Signal Processing, vol. 55, no. 9, pp. 46834695, Sept. 2007.

[5] M. Gastpar and M. Vetterli, "Power, spatio-temporal bandwidth, and distortion in large sensor networks," IEEE J. Select. Areas Commun., vol. 23, no. 4, pp. 745-754, Apr. 2005.

[6] R. Knopp and P. A. Humblet, "Information capacity and power control in single-cell multiuser communications," in Proc. IEEE. Int. Conf. Commun., Seattle, WA, June 1995, pp. 331335.

[7] P. Viswanath, D. N. C. Tse, and R. Laroia, "Opportunistic beamforming using dumb antennas," IEEE Trans. Inform. Theory, vol. 48, no. 6, pp. 1277-1294, June 2002.

[8] X. Qin and R. A. Berry, "Distributed approaches for exploiting multiuser diversity in wireless networks," IEEE Trans. Inform. Theory, vol. 52, no. 2, pp. 392-413, Feb. 2006.

[9] Y.-W. P. Hong, K.-U. Lei, and C.-Y. Chi, "Channel-aware random access control for distributed estimation in sensor networks," IEEE Trans. Signal Processing, vol. 56, no. 7, pp. 2967-2980, July 2008.

[10] F. W. J. Olver, Asymptotics and Special Functions, Academic Press, New York, 1974.

[11] A. S. Leong and S. Dey, "On scaling laws of diversity schemes in decentralized estimation," Available at http://arxiv.org/abs/1002.4473.

[12] T. Berger, Z. Zhang, and H. Viswanathan, "The CEO problem," IEEE Trans. Inform. Theory, vol. 42, no. 3, pp. 887-902, May 1996.

[13] A. S. Leong, S. Dey, and J. S. Evans, "Asymptotics and power allocation for state estimation over fading channels," IEEE Trans. Aerosp. Electron. Syst., to appear. Available at http://arxiv.org/abs/0803.3850.

[14] A. J. Goldsmith and P. P. Varaiya, "Capacity of fading channels with channel side information," IEEE Trans. Inform. Theory, vol. 43, no. 6, pp. 1986-1992, Nov. 1997. 